\title{
Design of China Power Market Information Disclosure Mechanism Based on the Power System Reform
}

\author{
Yanzhang Sun ${ }^{1, a}$ Tao $\mathrm{LI}^{2, \mathrm{~b}, *}$ Yanping Wang ${ }^{1, \mathrm{c}}$ and Xuefei Deng ${ }^{1, \mathrm{~d}}$ \\ ${ }^{1}$ State Grid Shanxi Electric Power Company, State Grid Corporation of China (SGCC), Shanxi, \\ China; \\ ${ }^{2}$ School of Economics and management, North China Electric Power University, Changping District, \\ Beijing, China. \\ aswws2008@163.com, b,*|t@ncepu.edu.cn, cycgdwyp@163.com, dsxsgcc@sohu.com
}

Keyword: Power market, information disclosure, information flow, power system reform.

\begin{abstract}
Since the new round of power system reform in China, there have been many achievements in the development of the power market. However, the relevant information on the current power market disclosure system is not yet perfect. The establishment of a complete information disclosure framework is an important part of China's power market construction, in this paper, power market information disclosure model and mechanism is designed on the basis of both theory and practice, so as to ensure that all kinds of information related to market transactions can be traced back and verifiable.
\end{abstract}

\section{Introduction}

China is currently in the wave of a new round of power system reform, which will lead the way through the relevant policy and practical experience to gradually establish a power market with low risk, active trading and complete functions, a fully competitive, open and orderly power market system is developing step by step. Information transparency has a crucial impact on market efficiency and orderliness, the improvement of information availability and transparency can push the market members to improve their behaviors and thus promote the healthy operation of the market. [1] Designs evaluation index to measure the effectiveness of power market information disclosure, and proposes a market transaction model which is suitable for domestic conditions applying above indicators. [2] Establishes a framework of interaction between market information and members' learning \& decision-making behavior, and simulates the evolution and equilibrium under different power market information disclosure system. [3] Points out the information disclosure on the national level resources to flow across regions as the core and that one on the provincial level which proves the credibility of the market operator and improves the provincial market efficiency should be established.

The establishment of a complete information disclosure framework is an important part of China's power market constructing, it is also conducive to establishing and perfecting the power market credit system, strengthening the construction of the market members credit system, standardizing the market order, making the information disclosure system and market credit system interrelated, so as to ensure that all kinds of information related to market transactions can be traced back and verifiable.

\section{Design of Power Market Information Disclosure Framework}

\subsection{Classification Method of Power Market Information.}

Several documents issued during the new round of power system reform in China mentioned the information classification method in the process of establishing information disclosure system. The main ideas are as follows: the market information is divided into public information, specific information and private information. Public information refers to data and other information that can be completely released publicly, specific information is publicly available data and other information 
to all market members, and private information is data and other information that a particular market member has access to and may not publish to other market members. Combined with the definition of relevant policy documents, a new information classification method will be proposed to help form a power market information data pool.

In accordance with the pertinence principle, combined with laws, regulations, market trading rules and regulatory requirements, in this paper, the power market information can be divided into disclose able and non-disclose able information, and the disclose able information can be divided into mandatory disclosure information and non-mandatory disclosure information.

\subsection{Disclosure Model of Power Market Information.}

\subsubsection{Direct Disclosure Model.}

Direct disclosure is the act that the power generation side, user side and power grid enterprises involved in the power market must disclose on the relevant platform according to the power market trading rules, that is, the members of the market directly disclose the information listed in the rules to the public. The content of the direct disclosure is mostly the basic information of the enterprise, the production parameters, etc. The main body of the disclosure model is the market member who discloses the information on its own, with the power generation enterprises, and mainly focuses on power generation enterprises, power users and power distribution companies.

\subsubsection{Indirect Disclosure Model.}

Indirect disclosure is an information disclosure model which is centrally disclosed by power trading institutions, and it is also important basis for establishing the theoretical framework of the power market information disclosure mechanism. Combined with the information needs analysis of all parties in the power market, it is not enough to use only the information from direct disclosure model to push the power market information disclosure mechanism achieve the effect of aiding members to make decisions. The information disclosed in the indirect disclosure model is mostly data that can be published, including macroeconomic information, industry development information, non-mandatory disclosure information required by members of the electricity market, transaction information generated by power trading organizations during providing services, etc. In addition, there are some non-disclosure information that affect the decision-making of market members. These data can be processed through mathematical tools to make the disclosure of non-disclosure information possible.

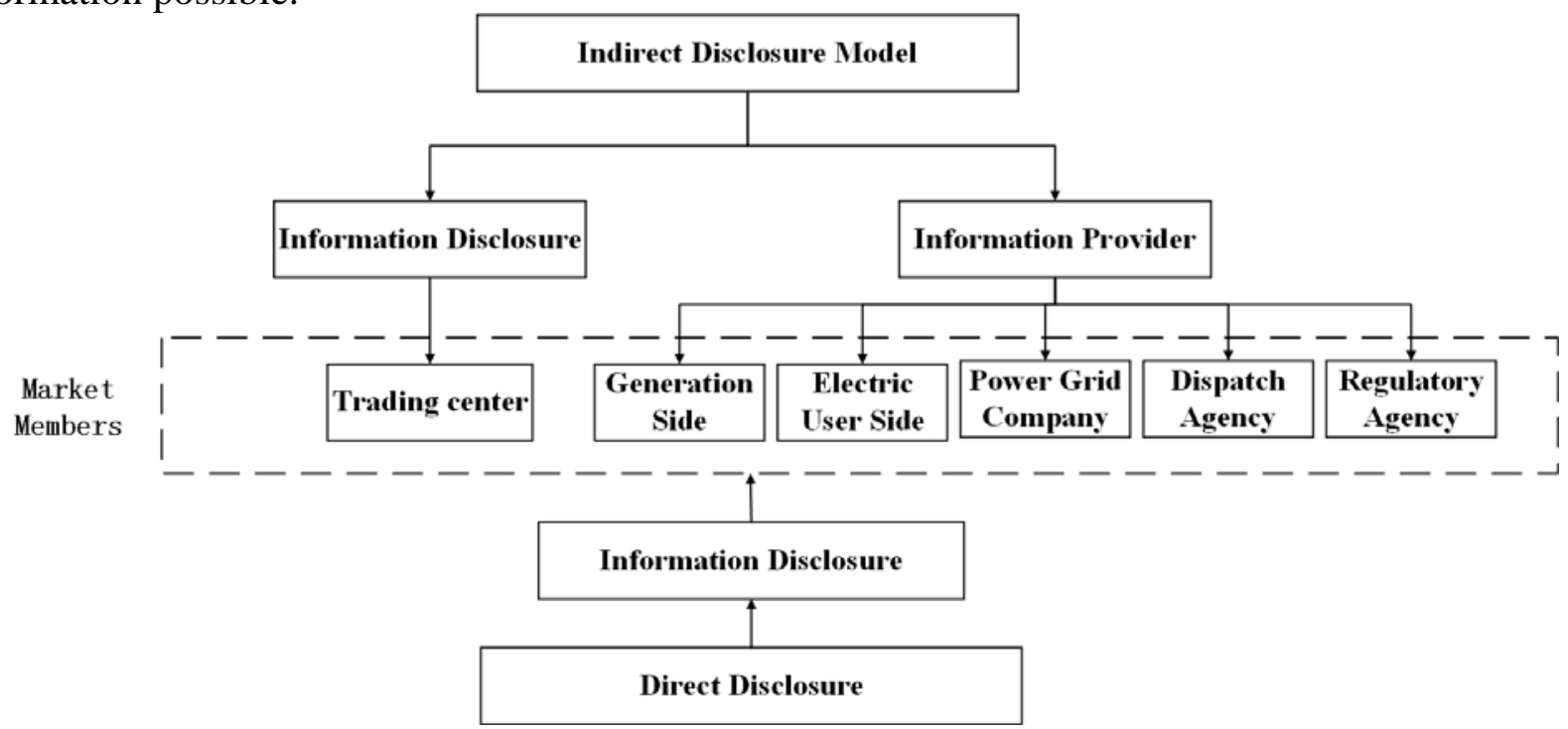

Figure 1. Disclosure Model of Power Market Information

\section{Design of Power Market Information Disclosure Mechanism}

By analyzing the transaction patterns, trading varieties and transaction flow of power market in various provinces and cities, summarizing and refining the theoretical knowledge of power market 
information disclosure, establishing a two-way power market information flow with power trading institutions as the core, and focusing on information supply and information disclosure. Numbers in the figure are the Information Flow (IF) generated during the disclosure process.

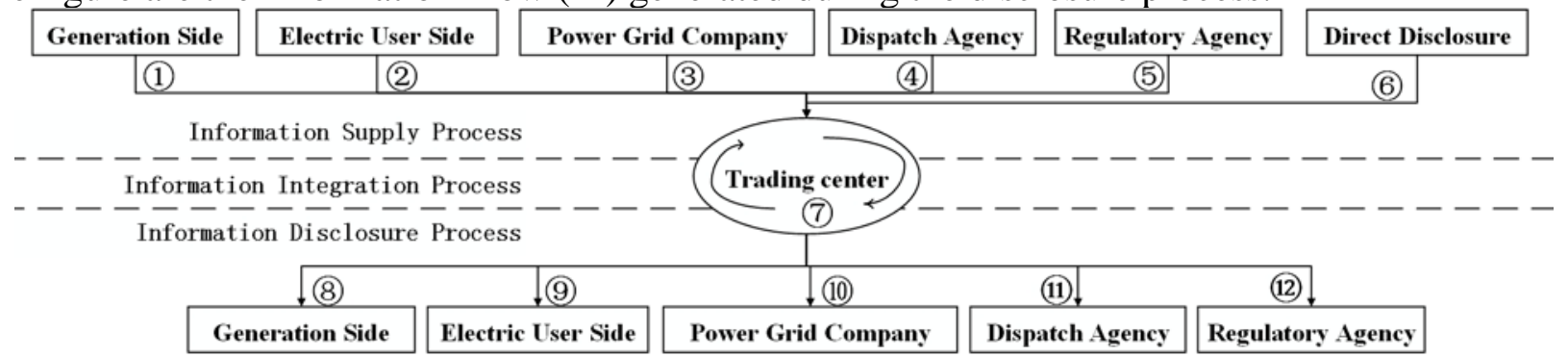

Figure 2. Information Flow of Power Market Disclosure Mechanism

\subsection{Information Supply Process.}

In order to ensure the fairness and effectiveness of the power market, the relevant parties shall provide all the information required by the rules to the trading center so as to standardize the market transaction behavior, which is conducive to the establishment of the power market information disclosure mechanism and also to the integrity of the power market system promotion.

IF1: Generation Side-Trading Center. Generation side submit business profiles, power generation capabilities, trading intentions and other information to the trading center.

IF2: Electric User Side-Trading Center. The user side, including sales companies and independent users, submit business profile, the status quo, electricity load, trading intentions and other information to trading center.

IF3: Power Grid Company-Trading Center. Power grid companies submit transmission and distribution plans, electricity supply and demand forecasting, electricity billing information and non-market information to trading center.

IF4: Dispatch Agency-Trading Center. The dispatch agency submits security constraints information, constrained clearing information, planning load information, etc.to trading center.

IF5: Regulatory Agency-Trading Center. The regulator provides relevant rules and regulations, proposes suggestions on power market construction, and urges the trading center to perform its service functions. Public documents will also be released to other market members at the same time.

IF6: Direct Disclosure Information-Trading Center. The trading center collects information disclosed by market members directly, which is helpful to reduce the workload of information collection.

\subsection{Information Integration Process.}

IF7: Trading Center Information Flow. The trading center in the power market is not only the information collector, but also the information producer. The trading center collects and integrates the information provided by all members and itself. By classifying information attributes, trading center can decide what information is not disclosed, what information is disclosed to market members, and what information is disclosed to the public.

\subsection{Information Disclosure Process.}

IF8: Trading Center -Generation Side. Through the centralized screening and processing of collected information, the trading center discloses market trading rules, transaction results and distribution service information to generation side.

IF9: Trading Center-Electric User Side. Through the centralized screening and processing of collected information, the trading center discloses market trading rules, transaction results and distribution service information to electric user side.

IF10: Trading Center- Power Grid Company. Through the centralized screening and processing of collected information, the trading center discloses market rules, market members' information and transaction information to the power grid company. 
IF11: Trading Center- Dispatch Agency. Through the centralized screening and processing of collected information, the trading center discloses market rules, user load information, transaction power forecasting, etc. to the dispatch agency.

IF12: Trading Center- Regulatory Agency. Through the centralized screening and processing of collected information, the trading center discloses the implementation of market rules, market transactions, credit rating of market members, etc. to the regulatory agency.

\section{Conclusion}

Since the new round of power system reform in China, there have been many achievements in the development of the power market. However, from the point of view of market members' transaction decision-making needs, or from the perspectives of relevant departments on the supervision of the operation of the power market, the relevant information on the current power market disclosure system is not yet perfect, the way of disclosure is relatively simple. Establishing a complete power market information disclosure mechanism will help reduce the cost of market information, improve the accuracy of disclosure information, ensure the timeliness of information disclosure, guarantee the fairness of information disclosure, and assist the market members in making scientific decisions.

\section{References}

[1]. Sun Key and Xia Qing. Effectiveness of Electricity Market Information Disclosure and Selection of Market Transaction, (Automation of Electricity Power Systems, 2008), 32(6):60-65.

[2]. Jiang Jian, Kang Chongqing and Xia Qing. Impacts of Information Mechanism on Electricity Market Equilibrium, (Automation of Electricity Power Systems, 2007), 31(6):11-16.

[3]. Ma Aiming, Zheng Haiyang, et al. Information Disclosure System in American Electricity Market and Its Enlightenment for China (Automation of Electricity Power Systems, 2017), 27(41): 49-57.

[4]. Zoo Peng, Chen Quoin, Xia Qing, He Chang, Ge Rue, Logical analysis of electricity spot market design in foreign countries and enlightenment and policy suggestions for China, (Automation of Electric Power Systems, 2014), 18-27.

[5]. Daria A, Mohamad A Z, Bayan H J D. Effects of Bidding Data Disclosure on Unilateral Exercise of Market Power (IEEE International Congress on Technology, Communication and Knowledge, 2016).

[6]. Zeng Ming, Liu Chao, Duane Xinhua, Li Na. The bilateral electricity market Modes of typical countries and our reference from America and Nordic, (East China Electric Power. 2013), 5-10. 